\title{
Le culte aux temps du Corona : la liberté de culte en période d'urgence sanitaire
}

\section{Frédéric Dieu}

\section{(2) OpenEdition}

1 Journals

Édition électronique

URL : https://journals.openedition.org/rdr/1465

DOI : $10.4000 /$ rdr. 1465

ISSN : 2534-7462

Éditeur

Presses universitaires de Strasbourg

\section{Édition imprimée}

Date de publication : 6 mai 2021

Pagination : 173-191

ISBN : 979-10-344-0089-8

ISSN : 2493-8637

Référence électronique

Frédéric Dieu, "Le culte aux temps du Corona : la liberté de culte en période d'urgence sanitaire », Revue du droit des religions [En ligne], 11 | 2021, mis en ligne le 06 mai 2021, consulté le 03 mai 2022. URL : http://journals.openedition.org/rdr/1465; DOI : https://doi.org/10.4000/rdr.1465

\section{(c) (†) (8)}

La revue du droit des religions est mise à disposition selon les termes de la Creative Commons Attribution - Pas d'Utilisation Commerciale 4.0 International - CC BY-NC 4.0. 


\section{Le culte aux temps du Corona : la liberté de culte en période d'urgence sanitaire}

\section{Frédéric DIEU}

Maître des requêtes au Conseil d'État

\section{INTRODUCTION}

L'un des livres du grand romancier colombien Gabriel Garcia Marquez s'intitule L'Amour aux temps du choléra, nous nous interrogerons dans cette chronique sur ce qu'est (devenu) le culte aux temps du Corona. Comme les autres libertés fondamentales (notamment celle d'aller et venir, celle d'entreprendre, celle d'accéder aux ouvres culturelles), la liberté de culte a été atteinte et fortement diminuée par le virus dit «Covid-19» qui a déjà franchi une décennie. Elle l'a été, à vrai dire, non par le virus lui-même, mais par l'état d'urgence décidé par le législateur et les mesures sanitaires prises par le Gouvernement pour ralentir et faire cesser sa propagation.

Nous connaissions déjà, certes, l'état d'urgence sécuritaire. Mais la particularité de cet état d'urgence sanitaire est qu'il s'impose à tous et non aux quelques-uns qui sont susceptibles de porter atteinte à la sécurité publique. Il est vrai que s'il s'impose à tous, c'est parce qu'il prétend bénéficier à tous, en protégeant toutes les «vies». Mais au nom de la protection de la vie, et d'une vie parfois réduite à son strict caractère biologique, il limite voire supprime les libertés qui font que la vie est la vie, qu'elle vaut d'être vécue et aimée, ne se réduisant pas à une subsistance qu'il s'agit de prolonger coûte que coûte.

Dans ce contexte, la liberté fondamentale de culte, peut-être plus fondamentale que les autres libertés fondamentales (en ce qu'elle maintient une 
transcendance en période de confinement et de désespérante horizontalité?), a souffert de très fortes limitations, dont la plupart des cultes semblent s'être accommodés. Le contentieux administratif relatif à ces limitations ${ }^{1}$, consacré en particulier à la contestation de l'interdiction des cérémonies religieuses dans les lieux de culte, a dès lors été très largement initié par des fidèles et des organisations catholiques. À deux reprises, le juge du référé-liberté leur a donné raison, peut-être parce que le temps était à la fin ou du moins à l'assouplissement du confinement. Demeure ainsi la question de savoir si, à l'avenir, de nouvelles périodes de confinement autoriseront des limitations aussi fortes de la liberté de culte.

\section{LA LIBERTÉ DE CULTE, QUELLE IMPORTANCE EN PÉRIODE D'URGENCE SANITAIRE?}

\subsection{LE CONTENTIEUX DE LA LIBERTÉ DE CULTE EN 2020 : BRÈVE CHRONOLOGIE}

\subsubsection{LES RECOURS REJETÉS}

\section{Défaut d'urgence du fait de l'urgence sanitaire}

Dans le cadre du premier confinement (le plus strict) du printemps 2020 (17 mars-11 mai 2020), le juge du référé-liberté, saisi de demandes de suspension de l'interdiction de pratiquer le culte dans les édifices du culte, a d'abord rejeté les recours au motif que la condition d'urgence posée par l'article L. 521-2 du Code de justice administrative (CJA) n'était pas remplie².

Ces recours étaient dirigés contre le décret no 2020-293 du 23 mars 2020 prescrivant les mesures générales nécessaires pour faire face à l'épidémie de Covid-19 dans le cadre de l'état d'urgence sanitaire, en particulier contre le IV de son article 8 interdisant tout rassemblement dans les lieux de culte, à l'exception des cérémonies funéraires dans la limite de vingt personnes.

Dans les ordonnances concernées, le juge des référés justifie le défaut d'urgence par deux éléments: en premier lieu, les «circonstances exceptionnelles aux vues desquelles le décret contesté a été pris et qui ont conduit le législateur à déclarer [...] l'état d'urgence sanitaire pour une durée de deux mois »; en second lieu, "l'intérêt public qui s'attache aux mesures de confinement prises, dans le contexte actuel de saturation des structures hospitalières».

1. Dont il faut souligner qu’il n'a donné lieu à aucune décision «fichée», c'est-à-dire dotée d'une autorité jurisprudentielle.

2. CE, réf., 24 mars 2020, nº 439694; 30 mars 2020, no 439809. 
Au fond, ces deux éléments, présentés de façon distincte par un «d'une part» et un « d'autre part», constituent un unique motif tiré de l'urgence sanitaire à laquelle le Gouvernement doit faire face, cette urgence étant caractérisée juridiquement (l'état d'urgence déclaré par le législateur) et concrètement (la saturation des hôpitaux). De façon sinon paradoxale du moins inattendue, cette urgence sanitaire semble donc, en elle-même, empêcher et anéantir l'urgence contentieuse s'attachant à la suspension des atteintes portées à la liberté fondamentale de culte, sans que le juge des référés ait à s'interroger sur la justification de ces atteintes au regard de la situation sanitaire.

Une telle motivation comporte un risque ou un biais: celui consistant à juger «irrecevables», pour défaut d'urgence, tous les référés-liberté (ou suspensions) dirigés contre des mesures motivées par une urgence sanitaire ou sécuritaire déclarée par le législateur et imposée par le Gouvernement, sans examen «au fond» de la nécessité et de la proportionnalité de ces mesures. Le risque, autrement dit, de permettre aux auteurs de ces mesures de s'affranchir de tout contrôle juridictionnel par l'affirmation d'une urgence qu'il est en leur pouvoir de définir, donc par l'énoncé d'une sorte de condition potestative.

\section{Nécessité et proportionnalité des restrictions apportées à la liberté de culte}

Ce sont peut-être la fragilité et le caractère quelque peu expéditif de cette solution qui ont conduit le juge du référé-liberté, dans un second temps, à rejeter «au fond» les recours dirigés contre l'interdiction de toute cérémonie religieuse dans les édifices du culte, sans se prononcer sur la condition d'urgence $^{3}$. Saisi, dans le cadre du «reconfinement » (ou plutôt du deuxième confinement) de l'automne 2020, d'une demande de suspension de l'exécution de l'article 47 du décret no 2020-1310 du 29 octobre 2020 à l'intitulé identique à celui du 23 mars 2020, le juge des référés a en effet estimé que l'interdiction de toute cérémonie religieuse dans les lieux de culte, à l'exception des cérémonies funéraires et des mariages ${ }^{4}$, était nécessaire et proportionnée ${ }^{5}$.

Quatre séries de considérations l'ont conduit à cette conclusion: la «tension» sur les structures hospitalières; l'interdiction concomitante de

3. CE, réf., 7 nov. 2020, n 445825 et a., Association Civitas et a.

4. On notera à cet égard l'office constructif et « réparateur» exercé par le juge du référé-liberté: malgré le silence des dispositions en cause, ou plutôt à cause de lui, le juge affirme en effet au point 14 de son ordonnance que «les cérémonies religieuses pour les mariages doivent être regardées, même si les dispositions gagneraient à être explicitées, comme n'étant pas interdites dans les lieux de culte, dans la limite de six personnes, ainsi que l'a expressément indiqué le Premier ministre lors de sa conférence de presse du 28 octobre $2020 »$.

5. Solution réitérée par CE, réf., 19 nov. 2020, nº 446651. 
tous rassemblements de plus de six personnes dans tous les lieux ouverts au public; l'absence d'actualisation et d'adaptation des protocoles sanitaires élaborés lors du déconfinement pour les lieux de culte; enfin, le réexamen à venir des mesures contestées et «l'engagement à bref délai d'une concertation avec l'ensemble des représentants des principaux cultes », concertation dont le juge des référés a discrètement et habilement imposé la tenue ${ }^{6}$ en affirmant qu'elle était nécessairement impliquée par le réexamen annoncé par le Gouvernement.

Un tel contrôle au fond de la nécessité et de la proportionnalité des restrictions apportées à la liberté de culte aurait de toute évidence, et à plus forte raison du fait du caractère plus strict de celui-ci, conduit au rejet des recours présentés lors du premier confinement. Mais la solution retenue par le juge du référé-liberté dans son ordonnance du 7 novembre 2020 ne sera pas nécessairement transposable à d'éventuels prochains confinements, parce que les structures hospitalières ne seront pas nécessairement en voie de saturation et surtout parce que les lieux de culte ont élaboré et appliquent des protocoles sanitaires beaucoup plus stricts et à même d'empêcher la diffusion du virus lors des célébrations qui s'y déroulent.

Quant à l'argument du réexamen à venir des mesures de restriction, également mobilisé pour juger nécessaires et proportionnées ces mesures, il est à vrai dire d'assez peu de poids dans la mesure où l'obligation de réexamen périodique est posée par la loi elle-même qui prévoit que les mesures prises dans le cadre de l'état d'urgence sanitaire sont «strictement proportionnées aux risques sanitaires encourus et appropriées aux circonstances de temps et de lieu » et qu' «Il y est mis fin sans délai lorsqu'elles ne sont plus nécessaires» (CSS., art. L. 3131-15 et s.).

\section{Après la bataille: l'intervention du juge de l'excès de pouvoir}

La première décision du Conseil d'État relative à un recours pour excès de pouvoir formé contre les restrictions apportées à la liberté de culte est intervenue le 23 décembre $2020^{7}$. Le Conseil d'État a rejeté la demande d'annulation dirigée contre les mesures prises lors du premier confinement en s'appuyant sur quatre séries de considérations: le caractère clos des édifices du culte et les risques de propagation du virus durant les célébrations (du fait de la participation des fidèles: prières à haute voix, chants); la possible saturation des structures hospitalières; la pénurie de masques chirurgicaux

6. Sans prononcer expressément, dans le dispositif de son ordonnance, une injonction à l'égard du Gouvernement.

7. CE, 23 déc. $2020, n^{\circ} 439810$. 
et «FFP2»; enfin, le «caractère circonscrit dans le temps» de l'interdiction des rassemblements de plus de vingt personnes dans les lieux de culte et de l'interdiction des déplacements aux fins de pratiquer le culte.

Cette ultime considération présente cependant l'inconvénient, ou l'avantage $^{8}$, d'être presque toujours mobilisable pour rejeter la contestation des mesures de restriction. Surtout, l'on voit bien que le recours pour excès de pouvoir, du fait du délai dans lequel il est jugé, n'est pas adapté à la contestation des mesures restrictives de libertés fondamentales prises dans le cadre de l'état d'urgence sanitaire.

\subsubsection{LES ATTEINTES SANCTIONNÉES}

Sanction du maintien de l'interdiction générale et absolue des cérémonies cultuelles après la fin du premier confinement

Alors que venait de prendre fin le premier confinement, que s'amorçait donc le déconfinement et que s'annonçaient en outre plusieurs grandes fêtes religieuses, le juge du référé-liberté du Conseil d'État a été saisi d'une demande de modification de l'article 10 du décret $\mathrm{n}^{\circ}$ 2020-548 du 11 mai 2020, à l'intitulé identique à celui du 23 mars précédent et aux dispositions aussi restrictives que celles de son article 8 . Le juge a cette fois fait droit aux demandes présentées par diverses personnes et organisations de confession catholique, donnant au Premier ministre un délai de huit jours pour modifier les dispositions du III de l'article 10 du décret du 11 mai $2020^{9}$ afin de prendre les mesures strictement proportionnées aux risques sanitaires pour encadrer les rassemblements et réunions dans les lieux de culte ${ }^{10}$.

Dans cette ordonnance longuement motivée, quatre points retiennent l'attention.

Le premier est l'affirmation du caractère communautaire et situé, localisé, de la liberté de culte: «cette liberté ne se limite pas au droit de tout individu d'exprimer les convictions religieuses de son choix dans le respect de l'ordre public. Elle comporte également, parmi ses composantes essentielles, le droit de participer collectivement, sous la même réserve, à des cérémonies, en particulier dans les lieux de culte. La liberté du culte doit, cependant, être conciliée avec l'objectif de valeur constitutionnelle de protection de la santé. » Autrement dit, l'exercice de la liberté de culte par les fidèles d'une

\footnotetext{
8. Selon le point de vue, du requérant ou du Gouvernement, que l'on adopte.

9. Ce qui fut fait par le décret $n^{\circ} 2020-618$ du 22 mai 2020.

10. CE, réf., 18 mai 2020, $n^{\circ} 440366$ et a.
} 
religion consiste pour l'essentiel à se réunir dans leurs lieux de culte afin d'y participer aux cérémonies et célébrations qui s'y tiennent. Ce qui pose l'essence et la finalité liturgiques du culte.

Le deuxième point qui retient l'attention est celui affirmant que la condition d'urgence est remplie et qu'elle l'est pour deux raisons: parce qu'il demeure interdit de participer «en vrai», sur place, à des cérémonies religieuses non funéraires, alors que d'importantes fêtes «ont eu lieu au printemps dans les trois religions réunissant le plus grand nombre de fidèles en France» (il est donc question ici des fêtes déjà passées et non de celles à venir); et parce que la situation sanitaire s'est améliorée, ce qui a conduit au déconfinement.

Le troisième point notable est l'affirmation d'un risque sanitaire propre aux cérémonies se déroulant dans les lieux de culte et l'affirmation corrélative de la nécessité d'une réglementation sanitaire de ces cérémonies: «les cérémonies de culte $[\ldots]$ exposent les participants à un risque de contamination, lequel est d'autant plus élevé qu'elles ont lieu dans un espace clos, de taille restreinte, pendant une durée importante, avec un grand nombre de personnes, qu'elles s'accompagnent de prières récitées à haute voix ou de chants, de gestes rituels impliquant des contacts, de déplacements, ou encore d'échanges entre les participants, y compris en marge des cérémonies elles-mêmes [...]».

Enfin, l'ordonnance du 18 mai 2020 livre une analyse approfondie du caractère nécessaire et proportionné des mesures contestées, associant des arguments comparatifs (comparaison de rapprochement avec les activités soumises à des restrictions moindres: services de transport de voyageurs, commerces, établissements d'enseignement et bibliothèques; comparaison de distinction avec les activités soumises à des restrictions identiques: bars, musées, salles de spectacles, théâtres, cinémas...) à des arguments propres aux cérémonies cultuelles. Sur ce point, le juge des référés écarte l'existence d'un risque important et spécifique que manifesterait un "précédent» de février 2020 (le rassemblement évangélique organisé à Mulhouse) et estime qu'il est possible d'élaborer et de faire respecter des règles de sécurité adaptées aux cérémonies se tenant dans les lieux de culte, ce qui le conduit à juger disproportionnée l'interdiction générale et absolue de tout rassemblement ou réunion dans les établissements de culte, sous la seule réserve des cérémonies funéraires.

\section{Sanction de la jauge spécifique et absolue imposée aux lieux de culte}

Six mois après s'être vu enjoint de modifier les dispositions réglementaires interdisant toutes les cérémonies non funéraires dans les lieux de culte, alors que le deuxième confinement (30 octobre-15 décembre 2020) s'allégeait, le 
Premier ministre a de nouveau été sanctionné par le juge du référé-liberté du Conseil d'État, ce à la demande de personnes et d'organisations de confession catholique et évangélique, mais aussi, pour la première fois, de la hiérarchie de l'Église catholique (Conférence des évêques de France). C'est cette fois l'interdiction générale et absolue de toute cérémonie religieuse de plus de trente personnes qui a été jugée disproportionnée à la situation et aux risques sanitaires avancés par le Gouvernement pour la justifier ${ }^{11}$.

La sanction était à vrai dire assez prévisible puisque, comme le relève l'ordonnance, «aucune activité n'est soumise à une telle limitation indépendamment de la superficie des locaux en cause», de sorte que l'on était en présence d'une quasi-discrimination à motif religieux ou du moins d'un traitement discriminatoire des lieux de culte.

Comme dans son ordonnance du 18 mai 2020, le juge des référés du Conseil d'État, pour regarder comme remplie la condition d'urgence, se fonde sur le caractère général et absolu de la restriction en cause, qui prive de nombreux fidèles de la possibilité de participer aux cérémonies organisées dans les lieux de culte, ainsi que sur l'amélioration de la situation sanitaire. Il réaffirme en outre «la nécessité de réglementer [...] les conditions d'accès et de présence dans les établissements de culte».

Il juge ensuite disproportionnée l'interdiction de toute cérémonie religieuse de plus de trente personnes en l'absence de risque spécifique aux cérémonies cultuelles qui justifierait de leur imposer une jauge "personnelle» absolue alors que les autres établissements recevant du public se voient imposer une jauge relative dépendant du nombre de mètres carrés par personne ou d'un pourcentage de leur capacité d'accueil. Le juge des référés reprend en outre l'argument comparatif de distinction énoncé dans l'ordonnance du 18 mai 2020 en affirmant que les activités exercées dans les autres établissements recevant du public demeurant fermés (restaurants, bars, musées, salles de spectacles, théâtres, cinémas...) «ne sont pas de même nature» que celles exercées dans les lieux de culte, de même que les libertés fondamentales associées à ces activités respectives «ne sont pas les mêmes». Toujours sur le plan comparatif, l'ordonnance du 29 novembre 2020 juge enfin inopérante l'invocation des mesures «absolues» prises par les autres pays européens. Il se confirme donc que l'argument comparatif, qu'il soit invoqué par les requérants ou par le Gouvernement et qu'il soit accueilli ou écarté par le juge, tient une place importante dans le raisonnement tenu et les solutions adoptées par ce dernier.

11. CE, réf., 29 nov. 2020, no 446930 et a., Association Civitas et a. 


\subsection{VALEUR ET CONTENU DE LA LIBERTÉ DE CULTE}

\subsubsection{VALEUR DE LA LIBERTÉ DE CULTE}

\section{Une liberté plus fondamentale que les autres?}

Les ordonnances précitées le rappellent: la liberté de culte est, au sens de l'article L. 521-2 CJA, une liberté fondamentale ${ }^{12}$, de même que la liberté d'expression religieuse ${ }^{13}$. Il en va de même bien entendu de la liberté de religion ${ }^{14}$.

La liberté de culte est-elle en outre plus fondamentale que les autres libertés fondamentales? On pourrait le penser à la lecture des points 32 et 19 des ordonnances du 18 mai et du 29 novembre 2020. Examinant la proportionnalité au risque sanitaire de la mesure d'interdiction ou de forte limitation des cérémonies religieuses dans les lieux de culte, le juge des référés y affirme en effet que si d'autres établissements recevant du public (restaurants, bars, musées, salles de spectacles, théâtres, cinémas...) demeurent également fermés au public, «les activités qui y sont exercées ne sont pas de même nature et les libertés fondamentales qui sont en jeu ne sont pas les mêmes».

Affirmer cela, c'est, semble-t-il, juger que les restrictions visant les activités cultuelles, et les libertés fondamentales qui y sont associées, peuvent être plus importantes et plus «liberticides » que celles visant les autres établissements recevant du public. Et donc que la liberté d'entreprendre, de même que la libre communication des idées, la liberté de création artistique et «la liberté d'accès aux ouvres culturelles ${ }^{15}$ » peuvent souffrir des entorses plus larges et plus durables que la liberté de culte.

\section{Accès au culte et accès à la culture}

La lecture de l'ordonnance du 23 décembre 2020, relative à la contestation des dispositions de l'article 45 du décret $n^{\circ} 2020-1310$ du 29 octobre 2020 en ce qu'elles interdisent l'ouverture des salles de spectacles, des théâtres et des cinémas, est à cet égard édifiante.

Le juge des référés affirme certes que «le risque de transmission du virus, dans les établissements accueillant les spectacles vivants comme dans les cinémas, est plus faible que pour d'autres événements rassemblant du public en lieu clos» et qu'en conséquence le maintien de l'interdiction d'ouverture

12. CE, réf., 16 déc. 2004, $\mathrm{n}^{\circ}$ 264314: Lebon T.; 25 août 2005, $\mathrm{n}^{\circ}$ 284307, Commune de Massat: Lebon T.; 6 mai 2008, $\mathrm{n}^{\circ}$ 315631: Lebon T.

13. CE, réf., 9 nov. 2015, no 394333, Association musulmane El Fath et a.: Lebon T.

14. CE, réf., 29 juin 2006, no 294649: Lebon T.

15. CE, réf., 23 déc. 2020, no 447698 et a. 
ne serait nécessaire et proportionné à l'objectif de préservation de la santé publique «qu'en présence d'un contexte sanitaire marqué par un niveau particulièrement élevé de diffusion du virus au sein de la population susceptible de compromettre à court terme la prise en charge, notamment hospitalière, des personnes contaminées et des patients atteints d'autres affections ».

Mais c'est aussitôt pour juger que ce contexte est bien présent en l'espèce et que cela justifie de prolonger l'interdiction d'ouverture, alors que les lieux de culte, qui présentent un risque important de propagation du virus, demeurent ouverts aux fidèles et bien plus peuvent abriter des rassemblements. Comment, dans ces conditions, c'est-à-dire en présence d'un contexte et d'un risque sanitaires qui sont au moins aussi importants dans les lieux de culte que dans les lieux de culture, comment ne pas penser que la liberté fondamentale de culte et d'accès au culte est plus importante, et pour cette raison davantage protégée, que la liberté fondamentale d'accès à la culture? Et que cette dernière peut légalement souffrir une durable éclipse quand la première ne peut le tolérer?

Bien que la formulation présente dans les ordonnances du 18 mai et du 29 novembre 2020 ne soit guère explicite sur ce point (aucune hiérarchie n'est consacrée, seule une différence est affirmée), la question doit être posée, comme doit être interrogée la légitimité de cette différence de traitement. Car, du point de vue du service rendu et des bienfaits procurés à la personne et à la société, au regard du bien commun et de ce qui grandit l'homme et du moins donne un sens à sa vie et lui permet d'en affronter les épreuves, l'accès à la culture semble tout aussi vertueux et nécessaire que l'accès au culte et plus précisément aux cérémonies organisées dans les lieux de culte. Ce qui au demeurant plaiderait plutôt pour un «alignement vers le haut», c'est-àdire pour une ouverture des lieux de culture (même si certains demeurent accessibles ${ }^{16}$ ) aussi bien que des lieux de culte, d'autant que le risque de transmission du virus dans ces lieux est «plus faible que pour d'autres événements rassemblant du public en lieu clos».

\subsubsection{CONTENU DE LA LIBERTÉ DE CULTE}

\section{Une liberté qui s'exerce collectivement}

La notion de culte est assez familière au juge administratif, plus en tout cas que la notion de religion, plus large et «vaporeuse». Un avis d'Assemblée du

16. Dans le cadre du deuxième confinement, les librairies et bibliothèques étaient en effet ouvertes au public. 
Conseil d'État du 24 octobre $1997^{17}$ a défini le culte comme «la célébration de cérémonies organisées en vue de l'accomplissement par des personnes réunies par une même croyance religieuse de certains rites ou de certaines pratiques ${ }^{18}$. C'était déjà là affirmer le caractère communautaire, collectif, du culte: c'est à plusieurs que l'on pratique le culte et on le pratique préférentiellement dans des lieux de culte où l'on se rassemble à cette fin, de sorte que la liberté de culte comporte le droit de participer à la prière communautaire ${ }^{19}$.

C'est pourquoi cette liberté «ne se limite pas au droit de tout individu d'exprimer les convictions religieuses de son choix dans le respect de l'ordre public [mais] a également pour composante la libre disposition des biens nécessaires à l'exercice d'un culte ${ }^{20} »$. La Cour européenne des droits de l'homme juge également que le droit de manifester sa religion collectivement «se trouve vidé de toute substance» lorsqu'une "communauté religieuse ne peut disposer d'un lieu pour y pratiquer son culte ${ }^{21} \gg$.

Il est vrai que, pendant les confinements, les lieux de culte ont été dispensés de l'obligation de fermeture provisoire imposée à tous les établissements recevant du public à l'exception de ceux fournissant des biens ou services de première nécessité. Mais ce traitement préférentiel, s'il autorisait les fidèles à se rendre dans ces lieux pour y exercer à titre individuel leur liberté de religion, ne leur permettait pas d'y "participer collectivement » à des cérémonies. Or, aux termes des ordonnances du 18 mai et du 29 novembre 2020, il s'agit là de l'une des «composantes essentielles» de la liberté de culte.

\section{Composantes essentielles et composantes accessoires}

De nouveau, les termes utilisés par le juge des référés semblent recéler une hiérarchisation subliminale qui n'est plus celle différenciant la liberté de culte des autres libertés fondamentales, mais celle distinguant, au sein même de cette liberté, des composantes essentielles et des composantes accessoires ou secondaires.

17. CE, ass., avis, 24 oct. 1997, n 187122, Association locale pour le culte des témoins de Jéhovah de Riom: Lebon.

18. Tel n'est pas le cas de l'Union des athées qui regroupe «ceux qui considèrent Dieu comme un mythe»: CE, 17 juin 1988, $\mathrm{n}^{\circ}$ 63912, Union des athées: Lebon. En revanche, le culte krisnaite est un culte au sens de la loi de 1905: CE, 14 mai 1982, n 31102, Association internationale pour la conscience de Krisna: Lebon.

19. V. a contrario, ord. $\mathrm{n}^{\circ} 264314$, précit.

20. Ord. Commune de Massat, précit.

21. CEDH, 24 mai 2016, n 36915/10, Association de solidarité avec les témoins de Jéhovah et autres c. Turquie, § 90 . 
Que le droit de participer collectivement à des cérémonies dans des lieux de culte soit une composante essentielle de la liberté de culte est, il est vrai, logique et tout à fait conforme à l'objet du culte, à sa raison d'être, tels qu'ils sont définis par l'avis d'Assemblée du 24 octobre 1997. D'autant que le lieu de culte se voit attribuer, du fait de cet objet, un régime juridique spécifique et protecteur par rapport aux autres lieux publics et ouverts au public: c'est pourquoi le Conseil constitutionnel a assorti d'une réserve propre à ces lieux sa déclaration de conformité à la Constitution de la loi du 11 octobre 2010 interdisant la dissimulation du visage dans l'espace public, jugeant à cet égard que «l'interdiction de dissimuler son visage dans l'espace public ne saurait, sans porter une atteinte excessive à l'article 10 de la Déclaration de 1789, restreindre l'exercice de la liberté religieuse dans les lieux de culte ouverts au public ${ }^{22} »$.

Mais alors, peut-on distinguer des composantes accessoires ou secondaires au sein de la liberté de culte? À vrai dire, nous n'en voyons guère car celles qui pourraient être considérées comme telles ont en réalité plus à voir avec la liberté de religion qu'avec la liberté de culte proprement dite. Il en va ainsi notamment des prescriptions et observances religieuses, qu'elles soient alimentaires ou vestimentaires, voire physiques. À propos des premières, le Conseil d'État parle ainsi de «l'observance des prescriptions alimentaires résultant des croyances et pratiques religieuses ${ }^{23}$ », $\mathrm{d}^{\prime}$ « aliments proscrits par [les] convictions religieuses ${ }^{24} »$. À propos des secondes, il parle de signe d'appartenance religieuse ${ }^{25}$. C'est bien que tout cela ne se rattache pas, du moins pas directement, au culte et relève donc moins de la liberté fondamentale de culte que de la liberté fondamentale d'expression en matière religieuse, qui est une liberté fondamentale distincte de la première ${ }^{26}$.

Le qualificatif d'essentiel vise donc moins à poser une hiérarchie entre différentes composantes qu'à signifier l'importance et la valeur éminentes que revêt, au regard de la liberté de culte, la participation à des cérémonies religieuses dans les lieux de culte, tout simplement parce que cette participation constitue le cour de cette liberté, sans quoi elle serait lettre morte.

22. Cons. const., 7 oct. $2010, \mathrm{n}^{\circ} 2010-613$ DC, § 5 .

23. CE, 10 févr. 2016, no 385929: Lebon.

24. CE, 11 déc. 2020, n 426483, Commune de Chalon-sur-Saône: Lebon.

25. CE, avis, 3 mai 2000, $\mathrm{n}^{\circ}$ 217017: Lebon: à propos des signes portés par les fonctionnaires; CE, 5 déc. 2007, $\mathrm{n}^{\circ}$ 285394: Lebon: à propos des signes arborés par les élèves des établissements publics d'enseignement des premier et second degrés; 12 févr. 2020, $\mathrm{n}^{\circ}$ 418299: Lebon $T$. : à propos de la barbe comme signe d'appartenance religieuse.

26. CE, réf., 9 nov. 2015, no 394333, Association musulmane El Fath et a.: Lebon T. 


\section{LIMITATION ET CONTRÔLE LIMITÉ DES LIMITATIONS}

\subsection{UNE LIBERTÉ DONT LA LIMITATION EST PLUS OU MOINS CONSENTIE}

\subsubsection{UNE LIBERTÉ LIMITÉE}

\section{La nécessité d'une réglementation sanitaire des lieux de culte}

Chacune des ordonnances du juge du référé-liberté relative à la liberté fondamentale de culte énonce que cette liberté doit s'exercer dans le respect de l'ordre public et doit en particulier «être conciliée avec l'objectif de valeur constitutionnelle de protection de la santé».

Ce qui est un rappel, d'abord, de la loi du 9 décembre 1905, dont l'article $1^{\text {er }}$ dispose que la République «garantit le libre exercice des cultes [...] dans l'intérêt de l'ordre public » (c'est dire que si la République se mêle de culte, c'est parce que, et dans la mesure où, elle se doit d'assurer l'ordre public), davantage que de l'article 10 de la Déclaration des droits de l'homme et du citoyen qui ne vise pas, du moins pas directement, la liberté de culte, mais la liberté d'expression en matière religieuse ${ }^{27}$.

Ce qui est un rappel, ensuite, de la jurisprudence du Conseil constitutionnel qui fait de la protection de la santé un objectif de valeur constitutionnelle ${ }^{28}$, les commentaires des décisions en cause paraissant d'ailleurs assimiler la protection de la santé à la protection de la santé publique ${ }^{29}$, alors que la jurisprudence du Conseil constitutionnel semble distinguer l'objectif constitutionnel de protection de la santé du principe de valeur constitutionnelle que constitue l'«impératif de protection de la santé publique ${ }^{30}$.

Quoiqu'il en soit, devant ainsi être concilié avec l'objectif constitutionnel de protection de la santé, l'exercice de la liberté de culte, et en particulier sa composante essentielle qu'est la participation à des cérémonies religieuses dans les lieux de culte, est en réalité devenu fortement dépendant de la situation sanitaire. En période de confinement strict, comme celui qui fut appliqué du 17 mars au 11 mai 2020 et qui s'étendait à la plupart des commerces et établissements d'enseignement, la liberté de culte, dans cette composante, cède devant la protection de la santé et disparaît totalement.

27. «Nul ne doit être inquiété pour ses opinions, même religieuses, pourvu que leur manifestation ne trouble pas l'ordre public établi par la loi.»

28. Cons. const., 13 août 1993, no 93-325 DC, § 70; 18 déc. 2014, n 2014-706 DC, § 25; 31 janv. 2020, n 2019-823 QPC, \& 5.

29. V. à cet égard le commentaire de la décision QPC du 31 janvier 2020, précit., p. 12-13.

30. Cons. const., 8 janv. 1991, nº 90-283 DC, § 29. 
Pour le dire autrement, l'exercice de la liberté de culte est jugé incompatible avec l'objectif de protection de la santé. Au regard de l'importance qui est la sienne de l'aveu même du juge, cela peut paraître excessif. L'on peut cependant penser que si un confinement aussi strict devait être de nouveau décidé et appliqué, les lieux de culte pourraient continuer d'accueillir des cérémonies en se prévalant des protocoles sanitaires éprouvés mis en place depuis plusieurs mois. À l'inverse, en période de confinement plus lâche et plus ciblé, moins général, l'exercice de la liberté de culte est compatible avec l'objectif de protection de la santé, sous réserve de la mise en œuvre dans les lieux de culte d'un protocole sanitaire adapté.

Cette obligation, rappelée dans toutes les ordonnances rendues par le juge du référé-liberté, ne fait plus débat en effet: les conditions d'accès et de présence dans les lieux de culte doivent faire l'objet d'une réglementation sanitaire qu'il incombe au desservant de faire respecter, sous le contrôle de l'Administration, plus particulièrement du préfet, et sous peine de fermeture du lieu concerné. Il a ainsi été jugé que l'obligation du port du masque dans les lieux de culte ne porte pas atteinte à l'exercice de la liberté de culte ${ }^{31}$.

\section{Une évolution de l'équilibre «institutionnel» en matière de police des lieux de culte}

La nécessité d'une réglementation sanitaire des lieux de culte conduit toutefois à modifier l'équilibre «institutionnel » existant en matière de police de ces lieux. À la séparation habituelle des fonctions entre l'affectataire cultuel et le maire de la commune d'implantation du lieu de culte s'ajoute en effet une relation, voire une collaboration, entre l'affectataire cultuel et l'administration locale de l'État (préfet, voire agence régionale de santé).

Traditionnellement, et cette répartition des rôles demeure d'ailleurs en période d'urgence sanitaire même si elle passe au second plan, il revient au seul ministre du culte desservant l'édifice cultuel d'en régler l'usage afin, bien sûr, d'organiser l'exercice du culte: il a «la garde et la police de l'église en vue d'assurer aux fidèles l'exercice de leur religion» et il est seul chargé de «régler l'usage» du lieu de culte qu'il dessert ${ }^{32}$. L'affectataire dispose ainsi, seul, d'un pouvoir d'organisation et de police du culte à l'intérieur de l'édifice cultuel qui lui est affecté $e^{33}$.

31. CE, réf., 12 juin 2020, no 440840.

32. Ord. Commune de Massat, précit.

33. C'est donc tout l'ordre intérieur qui relève du desservant et c'est l'ensemble de l'intérieur de l'édifice qui est sous sa seule responsabilité. Concrètement, c'est donc au seul desservant qu'il appartient de fixer les horaires des célébrations et plus largement les horaires 
Le maire, qui est pourtant l'exécutif de la collectivité propriétaire de l'édifice, est ainsi privé de tous pouvoirs à l'intérieur de l'édifice cultuel (construit avant 1905), sauf s'il s'agit d'assurer le respect de l'ordre public, notamment de la sécurité publique ${ }^{34}$ ou de régler les sonneries civiles sur le fondement de l'article 27 de la loi de $1905^{35}$.

Très protectrice de ce droit de jouissance exclusif de l'édifice cultuel du desservant, la jurisprudence du Conseil d'État a pu affirmer qu'aucune décision ne pouvait «avoir indirectement pour effet d'enlever au ministre du culte occupant l'église le droit d'en régler l'usage ${ }^{36} »$. L'exclusivité ainsi accordée à l'affectataire cultuel dans l'organisation du culte au sein de l'édifice cultuel, et l'étendue des pouvoirs qui lui sont accordés dans ce cadre, sont toutefois «finalisées», c'est-à-dire affirmées et garanties afin de «faire respecter la destination cultuelle de l'édifice, voulue par la loi, et de réglementer en conséquence l'utilisation de l'église et de son mobilier ${ }^{37} \%$. La première fonction de l'affectataire cultuel est donc «la protection de la destination cultuelle ${ }^{38}$ », ce pour quoi il ne peut lui être imposé «aucune obligation de caractère matériel à l'intérieur de l'église, telle qu'une obligation de sécurité qui tendrait à l'assimiler à l'exploitant d'un lieu ouvert au public ou à une collectivité publique administrant un bien du domaine public ${ }^{39} »$.

Et précisément, l'urgence sanitaire liée à une pandémie conduit à investir l'État, et son représentant local le préfet, d'un pouvoir de police sanitaire qui s'exerce à l'égard de tous les lieux ouverts au public, parmi lesquels les lieux de culte. Comme l'écrit Jacques Fialaire ${ }^{40}$ : «Dans le cadre de l'état d'urgence sanitaire, une police administrative spéciale se met en place, dont les prérogatives sont partagées entre le Premier ministre, le ministre chargé

d'ouverture et de fermeture de l'édifice, d'organiser les cérémonies religieuses, d'assurer la police intérieure de l'édifice et plus généralement de régler l'utilisation de l'édifice et du mobilier qui la garnit ainsi que l'aménagement intérieur des lieux. Sur tous ces points, nous nous permettons de renvoyer à notre article: "Les prérogatives de l'affectataire cultuel », Revue du droit des religions, n 2, 2016, p. 139-156.

34. CE, 26 mai 1911, Ferry: Lebon, p. 638: menace d'effondrement de l'église. V. aussi CE, 26 déc. 1913, Abbé Lhuillier curé de Saint-Paterne à Orléans: Lebon, p. 1295.

35. CE, 24 févr. 1912, Abbé Sarralongue: Lebon, p. 250; 11 avril 1913, Abbé Sommé: Lebon, p. 392.

36. CE, 20 juin 1913, Abbé Arnaud: Lebon, p. 717.

37. Y. Gaudemet, Traité de droit administratif. Droit administratif des biens, t. 2, Paris, LGDJ, $14^{e}$ éd. 2012, p. 107.

38. Y. DÉliCAT, «Les pouvoirs de l'affectataire cultuel», AJDA 2012, p. 2103.

39. Cass. $2^{e}$ civ., 19 juill. 1966, SNCF et Dame Vautier c. Chanoine Rebuffat: Bull. no 812; V. sur ce point Circ., 29 juill. 2011, NOR: IOCD1121246C, \& 1.4.

40. «Liberté de culte et urgence sanitaire: les leçons de la jurisprudence», JCP A 2020, 2155. 
de la santé et les préfets de département. Mais les ministres du culte desservants, qui gardent leur pouvoir au titre de la police intérieure des lieux de culte, sont amenés à faire respecter les normes de distanciation sociale», le ministre du culte devenant ainsi «auxiliaire des autorités de l'État».

À cet égard, l'ordonnance précitée du 29 novembre 2020 énonce très clairement l'obligation qui pèse sur le desservant et le pouvoir de «substitution d'action» dont dispose le préfet lorsque les mesures mises en œuvre par le gestionnaire du culte sont absentes, insuffisantes ou inadaptées: «il incombe à chaque gestionnaire de lieu de culte de veiller au respect de la réglementation sanitaire, y compris lors de l'entrée et de la sortie de l'édifice $[\ldots]$ le préfet du département peut, après mise en demeure restée sans suite, interdire l'accueil du public dans cet établissements [sic] [...] si les conditions de leur organisation ainsi que les contrôles mis en place ne sont pas de nature à garantir le respect de cette réglementation».

Cette soumission du lieu de culte et de son desservant aux mesures sanitaires décidées par l'État et mises en œuvre par son représentant, et l'intrusion qui s'ensuit de ce dernier dans la police sanitaire du lieu de culte, constituent une évolution majeure au regard de l'équilibre institutionnel de séparation qui prévalait jusqu'à présent entre le desservant et le maire.

\subsubsection{UNE LIMITATION CONSENTIE?}

À l'exception de l'Église et des fidèles catholiques, la plupart des religions et de leurs fidèles semblent s'être accommodés des restrictions apportées à l'exercice du culte et en particulier de l'interdiction qui leur était faite de convier leurs fidèles à des célébrations religieuses.

Qu'ils soient protestants (à l'exception des évangéliques, requérants dans le recours ayant donné lieu à l'ordonnance précitée du 29 novembre 2020), juifs, musulmans, bouddhistes ou encore adeptes d'autres religions (songeons par exemple aux témoins de Jéhovah), les fidèles et leurs représentants n'ont pas contesté les mesures décidées par le Gouvernement. Les catholiques, leur Église, leurs organisations et leurs représentants ont ainsi porté seuls le fer contre ces restrictions, parvenant par deux fois à obtenir du juge qu'il contraigne le Gouvernement à lever les restrictions et concrètement à autoriser les célébrations religieuses dans les édifices du culte et donc les messes dans les églises.

À cet égard, cette période d'urgence sanitaire marque également une évolution, un nouveau moment, dans l'histoire du contentieux de la liberté de 
culte et de l'expression en matière religieuse. Opposant pour l'essentiel l'Église catholique à l'État dans les décennies qui ont suivi l'entrée en vigueur de la loi de Séparation, ce contentieux a été marqué, dans les décennies plus récentes, par un affrontement entre l'islam et l'État.

Or, les mesures de restriction décidées par le Gouvernement pour faire face à la crise sanitaire ont en quelque sorte replacé les catholiques et leur Église au premier plan du jeu contentieux: il est à cet égard remarquable que cette dernière, qui était restée en retrait dans un premier temps (lors du recours ayant donné lieu à l'ordonnance du 18 mai 2020), ait ensuite décidé de contester directement les mesures en cause, se joignant personnellement, via la conférence des évêques de France, aux auteurs du recours ayant donné lieu à l'ordonnance du 29 novembre 2020.

Autrement dit, en 2020, les catholiques se sont retrouvés à la pointe du combat pour la liberté de culte, ce qui s'explique probablement par le caractère essentiel qu'ils accordent à la messe et, plus spécifiquement, à la célébration de l'eucharistie: la religion catholique est en effet indissolublement liée à ce sacrement et au sacrifice du Christ dont il fait mémoire. Le culte catholique ne peut pas être seulement intime, personnel et domestique: il doit être extérieur, communautaire et ecclésial. Sa dimension et son assise sacramentelles, qui ne sont pas présentes dans les autres religions, même chrétiennes (à l'exception toutefois des Églises orthodoxes), fournissent probablement la cause de la contestation de mesures qui privent la plupart ${ }^{41}$ des fidèles de l'accès au sacrement de l'eucharistie ${ }^{42}$.

Il faut toutefois souligner que l'identité et la particularité des auteurs des recours sont sans influence sur la généralité des solutions rendues par le juge saisi de ces recours. Autrement dit, et puisque la justice est aveugle, le juge du référé-liberté saisi des recours formés par des catholiques a pris des décisions valant pour les fidèles de toutes les religions et pas seulement pour les catholiques: il en a été ainsi spécialement des décisions imposant au Gouvernement de lever l'interdiction générale et absolue des cérémonies non funéraires dans les lieux de culte. La généralité de la solution est ici comme l'envers du principe d'égal traitement des cultes: aucun culte ne pouvant

41. Car un accès individuel à l'eucharistie, rendu possible par la visite à domicile d'un ministre du culte catholique, mais dépendant de la disponibilité de celui-ci et donc limité, demeurait possible.

42. L'accès aux autres sacrements habituels (confession, sacrement des malades, mariage) n'a certes pas été interdit, mais le sacrement de l'eucharistie est central pour les catholiques, l'eucharistie étant, selon le catéchisme de l'Église catholique, "la source et le sommet de la vie chrétienne». 
bénéficier d'un traitement différent (qu'il soit favorable ou défavorable) de celui réservé aux autres cultes, le juge est forcé de rendre des décisions qui nuisent ou bénéficient en principe à tous les cultes.

À cet égard, le culte catholique, malgré son enracinement millénaire et singulier dans notre pays, ne peut juridiquement revendiquer un traitement préférentiel et, concrètement, bénéficier d'autorisations dont seraient privés les autres cultes. Il n'empêche qu'en se portant seul au front contentieux pour réclamer la liberté de célébrer la messe communautaire dans les églises, et en obtenant par deux fois que le Gouvernement renonce aux interdictions et restrictions qu'il avait décidées sur ce point, le culte catholique a clairement manifesté sa singularité et sa combativité par rapport aux autres cultes.

\subsection{LA QUESTION DES VOIES DE RECOURS}

\subsubsection{DES RECOURS LIMITÉS}

Cela étant dit, force est toutefois de constater que la très grande majorité des recours en référé-liberté formés contre les mesures d'urgence sanitaire décidées par le Gouvernement a été rejetée par le juge ${ }^{43}$, notamment (nous l'avons vu au début de cette chronique) pour défaut de satisfaction de la condition d'urgence posée par l'article L. 521-2 CJA ${ }^{44}$. Cela à l'issue d'un raisonnement quasi-circulaire et en tout cas expéditif consistant à juger non remplie cette condition dès qu'il existe une urgence sanitaire, alors précisément que le recours vise à contester les mesures prises au nom de cette urgence. Raisonnement quasi-circulaire et expéditif qui, en tant qu'il fait prévaloir l'intérêt public s'attachant à l'exécution (donc au refus de suspension) de la mesure contestée, conduit «à l'impossibilité pour les justiciables de [la] contester utilement ${ }^{45} »$, et fait ainsi de la voie de recours qu'est le référé-liberté une voie sans issue.

Il est vrai qu'une fois franchie la barrière de la condition d'urgence, le juge du référé-liberté se livre à un contrôle très serré des mesures contestées. Les ordonnances précitées du 18 mai et du 29 novembre 2020 démontrent

43. V. à cet égard N. Symchowicz, «État d'urgence sanitaire et contrôle juridictionnel des mesures de police», AJDA 2020, p. 2001.

44. Étant rappelé que le recours pour excès de pouvoir n'est d'aucune utilité eu égard au délai dans lequel il est jugé, qui le rend inutile face au caractère temporaire et du moins très évolutif des mesures décidées par le Gouvernement, de sorte que le juge de l'excès de pouvoir a toutes les «chances» de se prononcer sur des mesures qui n'ont plus cours et/ou qui ont été substantiellement modifiées lorsqu'intervient sa décision.

45. N. Symchowicz, art. cit. 
à elles seules que le référé-liberté peut être, pour ceux qui le forment, une voie de recours efficace. Et qu'en dépit des délais très brefs dans lesquels le juge rend son ordonnance, le contrôle de nécessité et de proportionnalité auquel sont soumises les mesures décidées par le Gouvernement est réel et même approfondi.

Mais encore faut-il la franchir, cette barrière de la condition d'urgence, et c'est sans doute dans l'appréciation plus ou moins stricte de cette condition, dans son appréciation variable selon les affaires, que réside la faiblesse de cette voie de recours. Car, d'une certaine manière, il est et sera toujours possible de juger que cette condition n'est pas satisfaite au regard de la situation d'urgence sanitaire et de l'intérêt public s'attachant à l'exécution des mesures prises pour y faire face. Ce qui conduit concrètement à soumettre le rétablissement de l'exercice des libertés fondamentales, parmi lesquelles la liberté de culte, à l'amélioration de la situation sanitaire telle qu'elle est exposée par le Gouvernement et les diverses autorités sanitaires, le juge ne disposant pas de données qui lui sont propres. Et ce qui, en conséquence, conduit théoriquement à autoriser la suspension de ces libertés aussi longtemps que cette amélioration ne se manifeste pas. On peut douter de la légitimité d'une telle suspension durable et de son acceptabilité sociale.

En tout état de cause, et afin que le juge du référé-liberté puisse exercer un contrôle «au fond» des mesures prises, l'on peut souhaiter que la condition d'urgence posée par l'article L. 521-2 CJA soit appréciée de manière plus souple par le juge du référé-liberté.

\subsubsection{DES SOLUTIONS CONSTRUCTIVES}

Signalons pour terminer, et pour nuancer notre appréciation critique du référé-liberté, que le juge saisi d'un tel recours peut exercer son office de façon constructive et pédagogique, en réparant certains «oublis» dans les textes contestés ou en énonçant à l'adresse des requérants les libertés dont ils disposent.

C'est ainsi que dans l'ordonnance précitée du 7 novembre $2020^{46}$, le juge du référé-liberté, saisi d'une contestation du décret du 29 octobre 2020 et des mesures de «reconfinement», précise que «les cérémonies religieuses pour les mariages doivent être regardées, même si les dispositions gagneraient à être explicitées, comme n'étant pas interdites dans les lieux de culte», ce qui

46. CE, réf., 7 nov. 2020, no 445825 et a., Association Civitas et a. 
conduit à maintenir l'autorisation des mariages religieux dans les lieux de culte, même si, limitées à six personnes, ces cérémonies ont probablement été peu nombreuses.

Dans l'ordonnance précitée du 19 novembre $2020^{47}$, le juge du référéliberté a par ailleurs énoncé qu'il n'était pas «établi que le ministre de l'intérieur aurait adressé à ses services des instructions tendant à ce que soit interdite, par principe, toute manifestation sur la voie publique pouvant, par son but ou par sa forme, être regardée comme la manifestation extérieure d'un culte». L'on comprend donc que, selon le juge des référés, la liberté de manifestation sur la voie publique, qui a quant à elle toujours été maintenue, à la différence de la liberté de réunion et de rassemblement, bénéficie aux fidèles d'un culte. Mais l'on peut s'interroger sur cette notion de «manifestation extérieure d'un culte»: s'agit-il d'autoriser la pratique du culte sur la voie publique (par exemple l'organisation de messes en plein air, celle de prières, de processions) ou s'agit-il simplement d'autoriser la tenue de manifestations de fidèles revendiquant et réclamant le rétablissement total de la liberté de culte sans que les participants soient autorisés à saisir l'occasion de cette manifestation revendicative pour pratiquer leur culte?

Cette seconde interprétation a beau être la plus conforme à l'«esprit» des mesures décidées par le Gouvernement, la première demeure possible au regard de la rédaction retenue par le juge des référés. 